\title{
Netbench - large-scale network device testing with real-life traffic patterns
}

\author{
Stefan Nicolae Stancu ${ }^{1, *}$, Adam Lukasz Krajewski ${ }^{1}$, Mattia Cadeddu ${ }^{2}$, Marta Antosik ${ }^{1}$, and \\ Bernd Panzer-Steindel ${ }^{1}$ \\ ${ }^{1}$ CERN, Geneva Switzerland \\ ${ }^{2}$ Università degli Studi di Cagliari (IT)
}

\begin{abstract}
Network performance is key to the correct operation of any modern data centre infrastructure or data acquisition (DAQ) system. Hence, it is crucial to ensure the devices employed in the network are carefully selected to meet the required needs. Specialized commercial testers implement standardized tests [1,2], which benchmark the performance of network devices under reproducible, yet artificial conditions. Netbench is a network-testing framework, relying on commodity servers and NICs, that enables the evaluation of network devices performance for handling traffic-patterns that closely resemble real-life usage, at a reasonably affordable price. We will present the architecture of the Netbench framework, its capabilities and how they complement the use of specialized commercial testers (e.g. competing TCP flows that create temporary congestion provide a good benchmark of buffering capabilities in real-life scenarios). Last but not least, we will describe how CERN used Netbench for performing large scale tests with partial-mesh and full-mesh TCP flows [3], an essential validation point during its most recent high-end routers call for tender.
\end{abstract}

\section{Introduction}

When evaluating the performance of network devices we need to ensure they have the required features in terms of management and protocols (software features, with a fast enhancement cycle) and, more importantly, in terms of raw performance (hardware features, with a much slower enhancement cycle).

Given the diverse nature of CERN's networks (campus network, data centre, accelerator control network, experiments' control and data acquisition networks), we need to ensure that devices can correctly cope with various traffic patterns. For example:

- A full-mesh traffic distribution [3] is most likely the best benchmarking pattern for a network device to be employed in a multi-purpose data-centre;

- A unidirectional partial-mesh traffic distribution [3] closely mimics the event-building traffic pattern from a DAQ system.

The established benchmarking methodology $[1,2]$ consists of various tests that create pre-defined traffic patterns, in order to allow a precise comparison. However, some features

*e-mail: stefan.stancu@cern.ch 
(e.g. buffer depth) are not exercised by these tests, and performing a full test for a high end device with many ports requires a large number of expensive commercial testers.

We will start by presenting the typical approach to benchmarking network devices using specialized commercial testers, and describe the benefits and limitations of these approaches. Subsequently we will introduce Netbench (the network testing framework that we have developed, which uses commodity servers and the iperf3 [4] traffic generator) and describe how it addresses the shortcomings of specialized commercial testers (i.e. the cost of large-scale setups, and the ability to inject realistic traffic patterns that exercise buffering capabilities). Furthermore, we will present how CERN has successfully benchmarked high-end routers using an affordable combination of a small scale setup with specialized commercial testers (two ports) and a Netbench setup with 64 servers for large scale traffic injection.

\section{Benchmarking with specialized commercial network testers}

In this section we will present the two typical approaches for benchmarking network devices performance using dedicated network testers: the full test approach (desirable, but costly, due to the high tester port count requirement) and the "snake-test" approach (affordable, but limited to a simple linear traffic pattern).

\subsection{Full test with specialized network testers}

The complete benchmark of a device under test (DUT) requires connecting a tester port to each port of the DUT, as depicted in figure 1. The full mesh test described in [2] exercises the switching fabric of the DUT, but does not stress-test its buffers, because each tester port must choose its destination in a round-robin fashion that is meant to avoid congestion (i.e. two ports should never simultaneously send packets to the same third port). Specialized congestion tests are foreseen, but they only exercise a handful of ports.

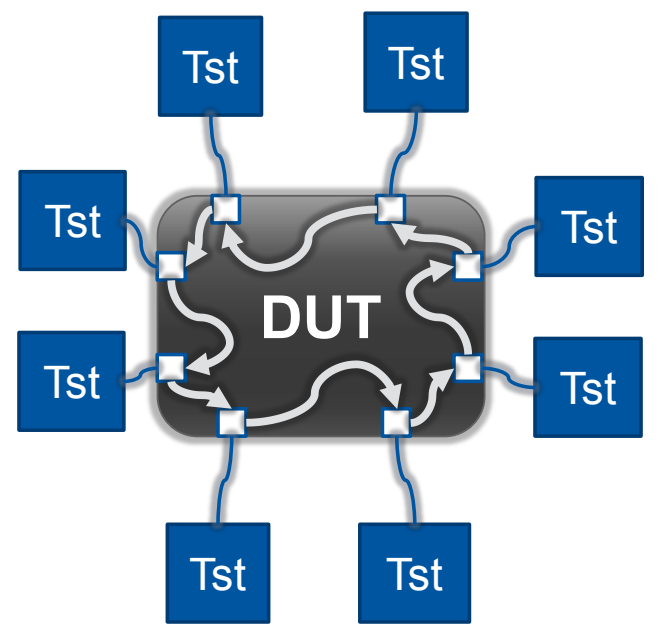

Figure 1. Full DUT benchmarking using commercial testers.

The full test with specialized network testers has the following advantages:

- It fully exercises all the ports, at line-rate and with a fair range of packet sizes.

- It enables sending complex traffic distributions through the DUT, notably partial-mesh and full-mesh.

The shortcomings of this test setup are: 
- The cost is high, due to the large number of required tester nodes ${ }^{1}$. Benchmarking a high end device with hundreds of ports (e.g. 100 Gigabit Ethernet ports) is cost prohibitive for most companies. Such tests are generally performed by specialized third-party companies (e.g. [7]).

- The device buffering capabilities are only lightly exercised, due to the round-robin choice of destinations (as previously explained at the beginning of Sect. 2.1).

\section{2 "Snake test" with specialized network testers}

Due to the prohibitive cost of specialized network testers, most companies that need to benchmark network devices use only two tester ports to inject traffic into the device under test, and then repeatedly loop traffic back through all ports of the device (the "snake test", depicted in figure 2).

Benchmarking a DUT in a "snake-test" setup has the following advantages:

- It is an affordable test setup (it only requires two tester ports)

- It fully exercises all the port (line-rate traffic for any packet size)

The shortcomings of this test method are:

- It barely stresses the switching engine of the device, as the traffic follows a linear path. The simplest approach is to use a sequential path (as illustrated in figure 2a), with the disadvantage of being easy to predict and optimize on the manufacturer side, and barely exercising inter-module communication for modular devices. A better approach is to choose a random linear path (as depicted in figure $2 \mathrm{~b}$ ), because such a path is impossible to predict and optimize, and it better exercises the inter-module communication for modular devices.

- The buffering capabilities of the DUT are not exercised, as the linear path ensures a congestion free traffic pattern.

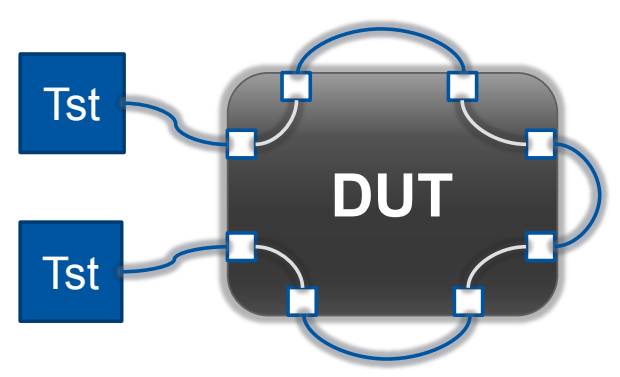

(a) Sequential path.

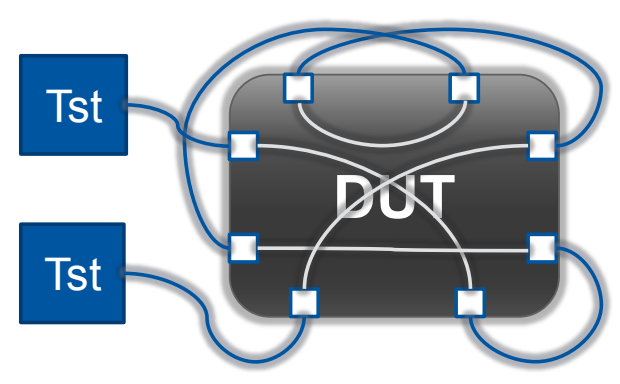

(b) Random path.

Figure 2: Snake test using commercial testers.

\footnotetext{
${ }^{1}$ Most established network testing equipment companies (e.g. [5] and [6]) produce generic network testers that perform a wide variety of protocol tests, on top of raw performance measurements. This complexity increases the per-port cost.
} 


\section{Netbench}

Netbench is a software platform that orchestrates traffic flow generation from multiple commodity servers (as illustrated in figure 3 ) and measures each flow's performance.

Benchmarking a DUT using Netbench addresses some of the previously mentioned shortcomings of specialized commercial testers (see Sect. 2) and offers the following advantages:

- It has a manageable cost. Since network device evaluation is typically a punctual event (e.g. a period of a few month), the servers can be time-shared between production and network tests.

- It possibly exercises all the ports of the device (the only limitation is the number of available servers)

- It can forward traffic with complex distributions (e.g. partial-mesh, full-mesh)

- It exercises the device buffering capabilities. The main traffic generation engine of Netbench is iperf 3 with the TCP protocol, and there is no synchronization between the senders. TCP flows compete freely for bandwidth and this competition creates congestion.

One of the drawbacks of Netbench with respect to dedicated appliances is the limited rate of packet generation on servers, which prevents line-rate tests for small frames on high speed links (e.g. 40 Gigabit Ethernet). However, real-life traffic is generally composed of a mixture of large and small frames (due to the nature of applications and protocols), so this limitation has little baring on assessing the performance of a DUT for real-life usage.

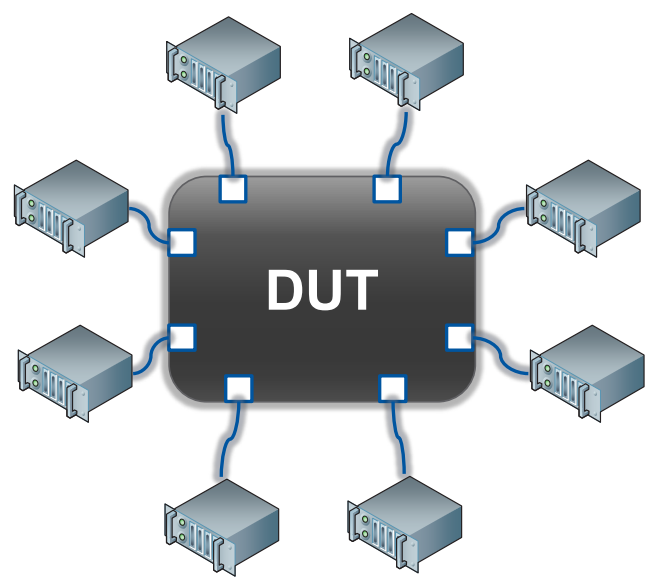

Figure 3. Netbench setup exercising all ports of a device.

In the rest of the section we will present the architecture and functionality of Netbench, as well as some sample results from the evaluation of high end routers.

\subsection{Netbench architecture}

As depicted in figure 4, Netbench has a layered architecture and uses standard technologies. At its core, it relies on iperf3 [4] as an engine to drive flows between servers ${ }^{2}$, but it can easily be extended to support other traffic generators. One of the advantages of using iperf3 is that it supports both IPv4 and IPv6, so Netbench can perform tests with both IP protocol versions. The orchestration platform that sets up multiple iperf3 sessions is written in Python and relies on XML-RPC for fast provisioning of flows. Per-flow statistics are gathered into a PostgreSQL database, and the results visualisation is based on a Python REST API and a web page using JavaScript and the D3.js library for displaying graphs.

\footnotetext{
${ }^{2}$ Currently Netbench uses TCP, but it is possible to adapt the tool for using other protocols supported by iperf3.
} 


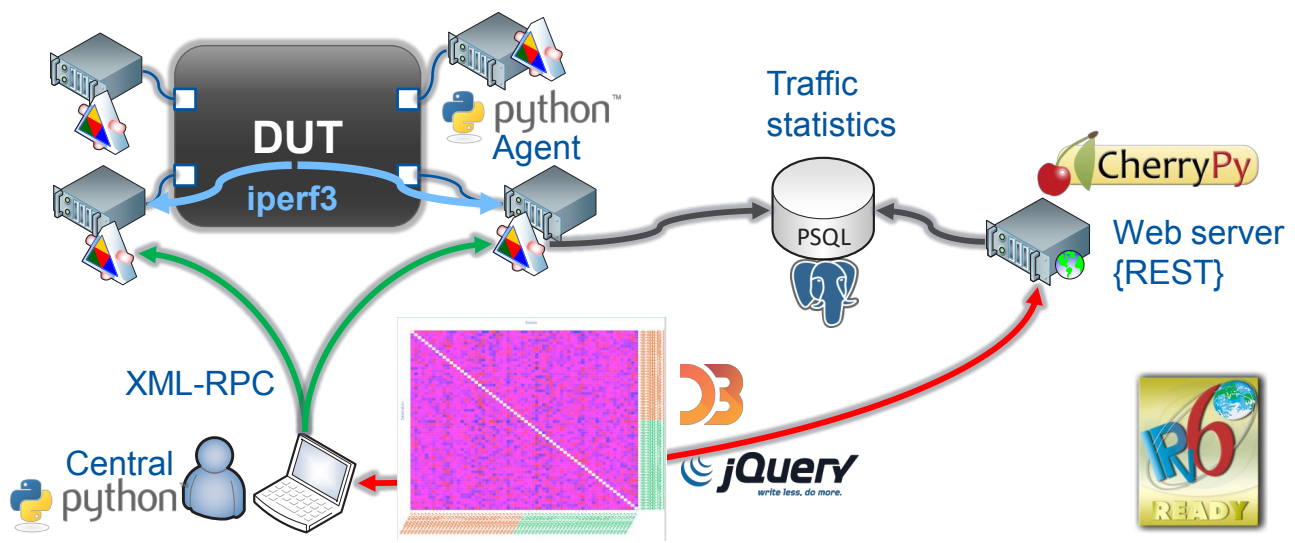

Figure 4: Netbench architecture.

Statistics are presented at different levels of detail allowing the human operator to quickly assess the overall state of a test. Figure 5 illustrates the available statistics for a full-mesh test with 64 nodes:

Flow count difference For each of the nodes taking part in the test we plot the difference between the number of flows that we expect to see on the node, and the number of flows recorded in the statistics database. For a test that is running successfully, the values for all nodes should be 0 (as illustrated in figure $5 \mathrm{a}^{3}$ ).

Per-node total bandwidth For each of the nodes, we plot the total transmit (Tx) and receive $(\mathrm{Rx})$ bandwidth. For a full-mesh test, and a DUT that handles flows in a fair manner, the total TX and RX bandwidth should be flat for all nodes (as illustrated in figure $5 b^{3}$ ).

Per-node flow bandwidth For each of the nodes, we compute the per-flow average bandwidth and standard-deviation for all the flows that are transmitted (Tx) or received (Rx) by the node. For a full-mesh test, and a DUT that handles flows in a fair manner, the mean bandwidth should be flat, and the standard deviation should be small. The results depicted in figure $5 \mathrm{c}^{3,4}$, show a deviation from this behaviour for a node that has a much higher standard deviation than the others.

Per-pair flow bandwidth A heat map for the bandwidth between each source and destination allows us to gain more insight into the behaviour of the device. For the sample test results, the heat map illustrated in figure $5 \mathrm{~d}^{5}$ clearly shows the origin of the previously observed abnormally high standard deviation: for the respective node, one flow uses much more bandwidth than all the others. Netbench is capable of instantiating multiple flows between each sender and destination. For such cases, a similar heat map is used to display the standard deviation of the flows for each source-destination pair.

\footnotetext{
${ }^{3}$ For each node there are two bars with different colors, one for Tx (transmit), one for Rx (receive).

${ }^{4}$ The standard deviation of a flow is represented as a red vertical line, superposed over the bar representing the average bandwidth of the respective flow.

${ }^{5}$ The average bandwidth of all flows between a given source and destination is colour-coded. The color bar at the bottom of the plot can be used to translate a colour into a bandwidth value.
} 


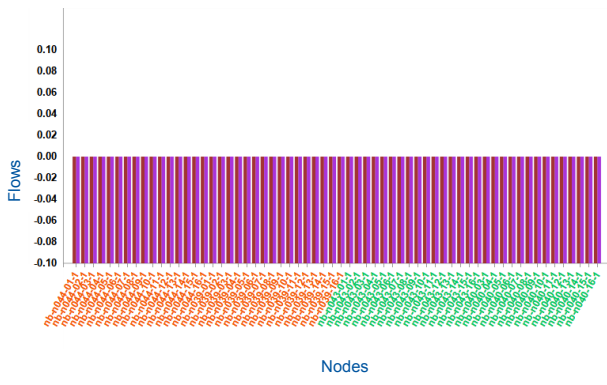

(a) Per-node flow count difference.

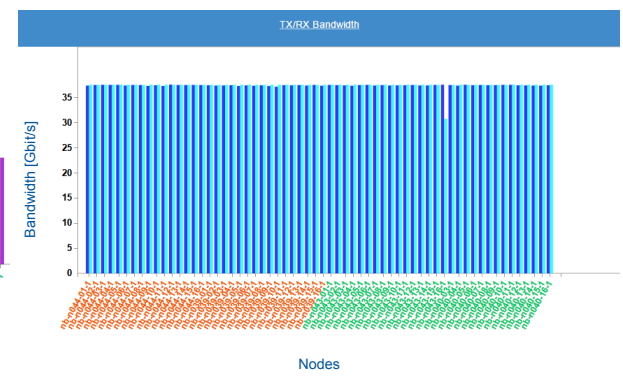

(b) Per-node total bandwidth.

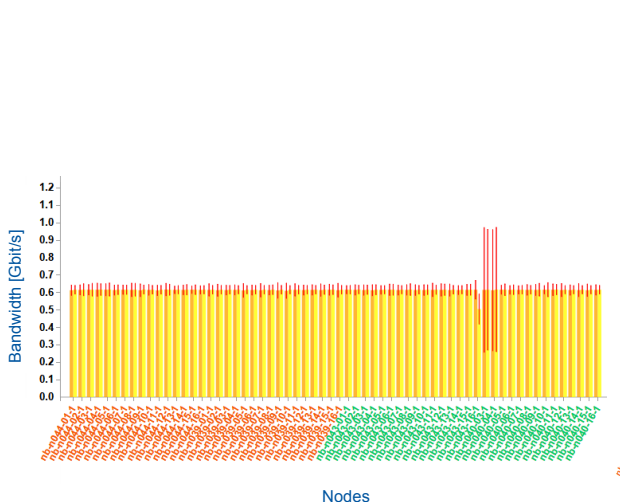

(c) Per-node flow bandwidth (average and stdev).

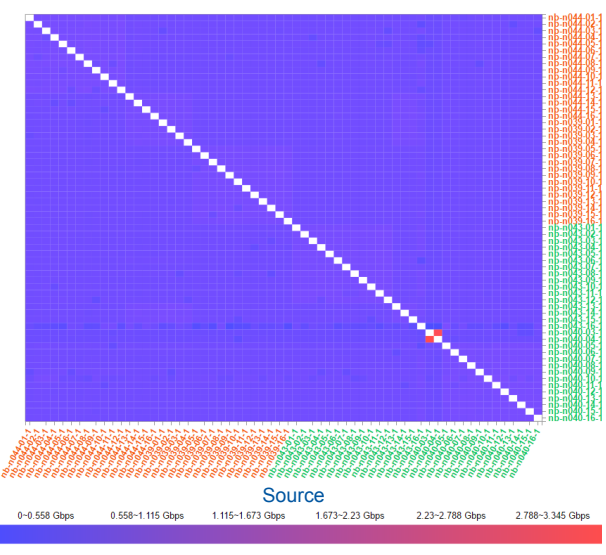

(d) Per-pair bandwidth heat map.

Figure 5: Netbench statistics plots with various level of detail.

\subsection{Netbench sample results}

During its last call for tender for high-end routers, CERN has employed Netbench ${ }^{6}$ for evaluating the behaviour of network devices when handling full and partial meshed TCP traffic. In this section we will present a sample result for a full-mesh test, that reveals Netbench's capability to create temporary congestion and exercise the buffering capabilities of network devices.

Figure 6a shows the results when running Netbench with TCP flows with an uncapped TCP window. It clearly shows two classes of behaviour. After interaction with the manufacturer, this was traced down to the internal architecture of the device and an uneven distribution of ports over internal modules (some modules had 12 tester ports connected, while others hand only 8 tester ports connected). The under-populated modules had more per-flow buffering capacity for inter-module transfers, compared to the fully-populated modules. Bigger buffers directly translate to a larger TCP round-trip time (RTT), and this results in less bandwidth for the flows (this is a know behaviour of most TCP congestion algorithms, and the measure of bandwidth imbalance as function of RTT is denoted as "RTT fairness").

Netbench with free running TCP window is practically emulating a large number of elephant flows. In practice, most flows are short lived and typically involve a series of transac-

\footnotetext{
${ }^{6}$ The setup comprised 64 servers equipped with 40 Gigabit Ethernet NICs
} 
tions. To emulate such real-life traffic patterns we capped the TCP window size ${ }^{7}$, and thus limited the amount of data transiting in the network. In these more realistic test conditions all flows received a fair share of the available bandwidth, as illustrated in figure $6 \mathrm{~b}$.

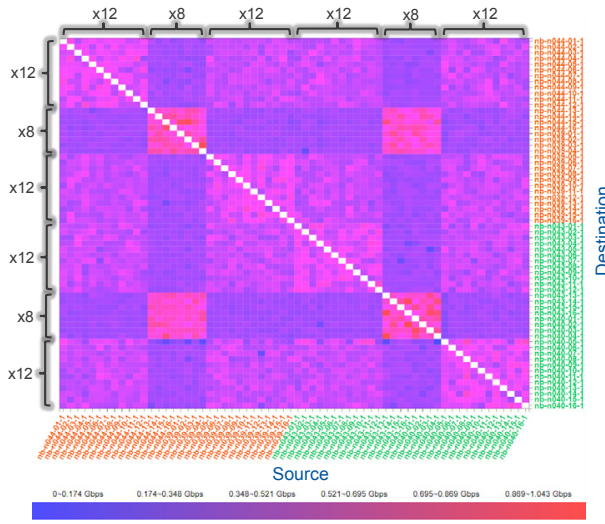

(a) Uncapped TCP window.

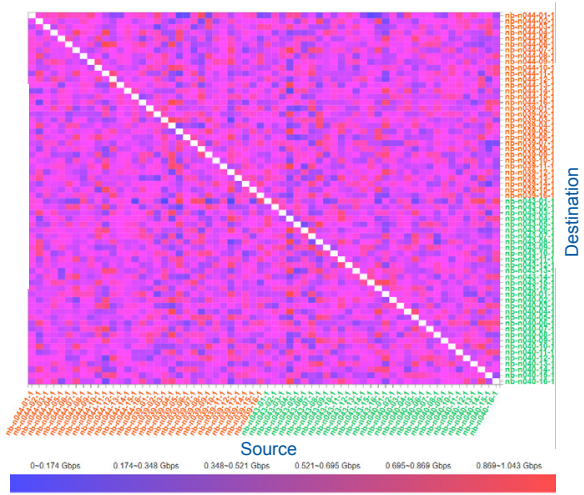

(b) TCP window capped to 64 kbyte.

Figure 6: Sample Netbench results from a full-mesh test.

\section{Conclusion}

We have developed Netbench, a tool that allows provisioning multiple TCP flows with various traffic distributions (pairs, partial and full-mesh). Table 1 summarizes Netbench's features in comparison to specialized commercial testers, and highlights its unique abilities (e.g. the potential to create large scale congestion and exercise the device buffering capabilities).

Table 1: Feature comparison between Netbench and specialized network testers.

\begin{tabular}{|l|l|l|l|}
\hline Feature & $\begin{array}{l}\text { Full test } \\
\text { (specialized testers) }\end{array}$ & $\begin{array}{l}\text { Snake test } \\
\text { (specialized testers) }\end{array}$ & Netbench \\
\hline Line-rate test & yes & yes & partial $^{*}$ \\
\hline Packet-size scan & yes & yes & no \\
\hline $\begin{array}{l}\text { Complex traffic patterns } \\
\text { (full or partial mesh) }\end{array}$ & yes & no & yes \\
\hline $\begin{array}{l}\text { Create congestion } \\
\text { (exercise buffering) }\end{array}$ & no & no & yes \\
\hline Cost for a large scale test & high & affordable & affordable** $^{* *}$ \\
\hline
\end{tabular}

* Only for large frames

** Assuming a time-shared usage of servers between production and network testing.

As detailed in Sect. 3.1, currently Netbench supports the orchestration of TCP flows and measures their bandwidth. Future work for enhancing Netbench should aim at measuring the

\footnotetext{
${ }^{7}$ The TCP window was limited to 64 kbyte (iperf3 -w 64k)
} 
latency between the nodes (e.g. using ping probes) and at supporting other protocols (e.g. UDP).

To summarize, we consider Netbench an essential complement to synthetic RFC tests $[1,2]$ performed with specialized commercial testers, as it enables affordable, large-scale testing of network devices with traffic patterns that closely resemble real-life conditions.

\section{References}

[1] S. Bradner, M. J., Benchmarking Methodology for Network Interconnect Devices, RFC 2544

[2] R. Mandeville, P. J., Benchmarking Methodology for LAN Switching Devices, RFC 2889

[3] R. Mandeville, Benchmarking Terminology for LAN Switching Devices, RFC 2285

[4] iperf3, http://software.es.net/iperf

[5] ixia, https://www.ixiacom.com/

[6] spirent, https://www. spirent.com/

[7] The Tolly Group, http://reports . tolly.com/AboutUs . aspx 